\title{
Title: EQUIVALENT CIRCUIT STUDY OF BEAM-LOADING USING A MOMENT METHOD
}

\author{
Author(s): Tai-Sen F. Wang \\ S. Machida \\ Y. Mori \\ C. Ohmori
}

\author{
LANSCE-1 \\ Institue for Nuclear \\ Study, University of \\ Tokyo \\ Institue for Nuclear \\ Study, University of \\ Tokyo \\ Institue for Nuclear \\ Study, University of \\ Tokyo
}

Submitted To: 1997 Particle Accelerator Conference

Vancouver BC, Canada

May 12-16, 1997

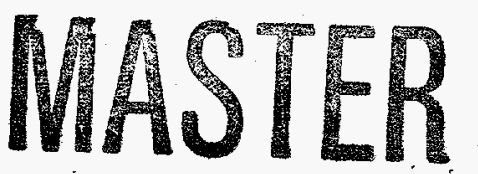

$$
\begin{gathered}
\text { PECEIVEO } \\
\text { JUL } 25 \text { भOT } \\
\text { OSTI }
\end{gathered}
$$

DISTRIBUTION OF THS DOCUMENT IS UNLMTED

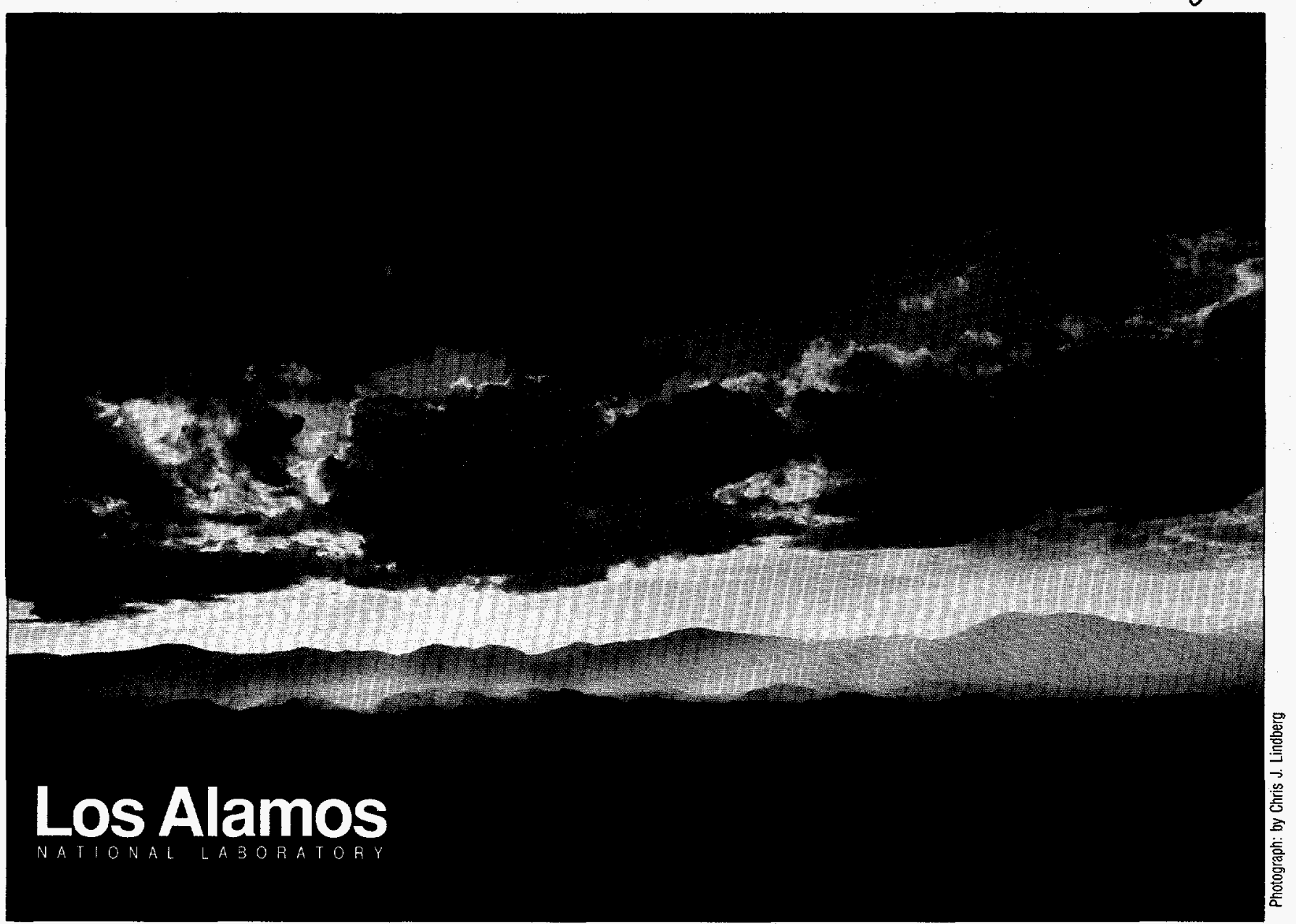

This is a preprint of a paper intended for publication in a journal or proceedings. Because changes may be made before publication, this preprint is made available with the understanding that it will not be cited or reproduced without the permission of the author. 


\section{DISCLAMIRR}

Portions of this doenment may be illegible in electronic image products. Images are produced from the best available original document. 


\section{DISCLAIMER}

This report was prepared as an account of work sponsored by an agency of the United States Government. Neither the United States Government nor any agency thereof, nor any of their employees, make any warranty, express or implied, or assumes any legal liability or responsibility for the accuracy, completeness, or usefulness of any information, apparatus, product, or process disclosed, or represents that its use would not infringe privately owned rights. Reference herein to any specific commercial product, process, or service by trade name, trademark, manufacturer, or otherwise does not necessarily constitute or imply its endorsement, recommendation, or favoring by the United States Government or any agency thereof. The views and opinions of authors expressed herein do not necessarily state or reflect those of the United States Government or any agency thereof. 


\title{
EQUIVALENT CIRCUIT STUDY OF BEAM-LOADING USING A MOMENT METHOD*
}

\author{
T. F. Wang, Los Alamos National Laboratory, Los Alamos, New Mexico 87545, USA \\ S. Machida, Y. Mori, and C. Ohmori \\ Institute for Nuclear Study, University of Tokyo, Tanashi, Tokyo 188, Japan
}

\section{Abstract}

In this work, we present a formalism by considering the perturbations in the moments of a bunched beam for the equivalent circuit model to include all harmonics of the synchrotron oscillation in a beam-cavity interaction system. The linear coupling among all longitudinal modes under the influence of a narrow-band impedance can be naturally incorporated in this new approach. We used this method to re-examine the coupling between the dipole and the quadrupole modes. The dispersion relation obtained by this new method was compared with that derived from the linearized Vlasov equation up to the second harmonic of the synchrotron motion. We found excellent qualitative agreements between these two approaches.

\section{INTRODUCTION}

In designing the radio frequency (rf) system of an accelerator or storage ring, the interaction between the charged particle beam and the rf cavity is often modeled by an equivalent circuit. Because of its simplicity and its convenience to incorporate with engineering designs, the applications of this kind of modeling can range from a simple estimation of power requirement to complicated studies of system stability and control designs. In a more elaborated approach, a beam-cavity interaction system can also be studied by using Vlasov-Maxwell equations to include the dynamics of particles in the beam. For example, the Robinson instability in circular machines was initially investigated via an equivalent circuit model and later on studied by using Vlasov equations.[1-3] One of the advantages of using the kinetic theory approach over the equivalent circuit model is that the coupling among-all synchrotron harmonics are covered in the formalism in a natural way. In all the equivalent circuit models utilized so far, only the dipole modes have been included. The reason is because a bunch of particles is modeled as a single macro-particle with no internal degree of freedom.

For narrow-band resonators, only those synchrotron sidebands near the resonant frequencies of the cavity contribute significantly to the beam-cavity interaction. It has been discussed previously, that for a tightly bunched beam interacting with a highly or moderately detuned narrowband resonator, the neglect of higher synchrotron harmonics is a good approximation. However, for long beam bunches or small cavity detuning, higher synchrotron harmonics may affect the stability appreciably; therefore, at least a few of the higher synchrotron harmonics should

\footnotetext{
* Work partially supported by US Department of Energy
}

be considered.[3] The purpose of this study is to formulate, at least in the linear regime, an approach in the equivalent circuit model that can take higher synchrotron harmonics into account. As will be discussed in the followings, one can incorporate any number of synchrotron harmonics in the equivalent circuit model by using the moment method.[4] A general moment method has been previously used to study transverse beam dynamics. $[5,6]$ The "moment" we will study here is the moment in the configuration space of a bunched beam, it does not include all the moments in the phase space as some previous studies did. For simplicity, we shall concentrate on the case below transition, and we limit our study to the coherent mode, or the " 0 " mode, stability of a multi-bunch system, i.e., the coupled-bunch modes are not considered at here.

\section{LINEARIZED EQUATIONS OF PARTICLE MOTION}

The equation of synchrotron motion of a beam particle is

$$
\frac{d^{2} \varphi}{d t^{2}}=\frac{\omega_{s}^{2}}{V_{s} \cos \psi_{s}}\left[V \sin \left(\psi_{s}+\phi_{\nu}-\varphi\right)-V_{s} \sin \psi_{s}\right]
$$

where $\varphi$ is the phase deviation of the particle's position with respect to the synchronous phase $\psi_{s}, t$ is the time, $\omega_{s}=\left[-q \eta h V_{s} \cos \psi_{s} /\left(2 \pi m_{0} \gamma R^{2}\right)\right]^{1 / 2}$, is the synchrotron frequency at equilibrium, $q$ and $m_{0}$ are the charge and the rest mass of a beam particle, respectively, $V_{s}$ is the maximum rf voltage on the cavity when the system is in steady state, $V$ is the voltage on the cavity, $\phi_{v}$ is the deviation of the voltage phase from its equilibrium, $\gamma$ is the ratio between the total energy and the rest mass of the synchronized beam particle, $h$ is the rf harmonic number, $\eta=\gamma_{t}^{-2}-\gamma^{-2}$, is the momentum slip factor for a machine with transition gamma $\gamma_{t}$, and $R$ is the effective machine radius. Assuming that $\varphi$ and $\phi_{v}$ are small quantities, $V=V_{s}+\hat{V}$ with $\hat{V} \ll<V_{s}$, Eq. (2.1) and its first order integral can be linearized to obtain

$$
\frac{d^{2} \varphi}{d t^{2}}+\omega_{s}^{2} \varphi \approx \omega_{s}^{2}\left(\phi_{v}+\frac{\hat{V}}{V_{s}} \tan \psi_{s}\right)
$$

and

$$
\left(\frac{d \varphi}{d t}\right)^{2} \approx-\omega_{s}^{2} \varphi^{2}-\omega_{s}^{2} \varphi^{2}\left(\frac{\hat{V}}{V_{s}}-\phi_{v} \tan \psi_{s}\right)+H_{0},
$$

where $H_{0}=\omega_{s}^{2} \varphi_{a}^{2}$, and $\varphi_{a}$ is the maximal excursion of the particle in the unperturbed if potential well. 


\section{THE MOMENTS AND THEIR EQUATIONS OF MOTION}

We assume that the phase space distribution of particles in a bunch, $f\left(z, v_{z}, t\right)$, is a steady part $f_{0}\left(z, v_{z}\right)$ plus a perturbation $f_{1}\left(z, v_{2}, t\right)$, i.e., $f=f_{0}+f_{1}$, where $z$ is the coordinate along the bunch length, and $v_{z}$ is the axial speed of a beam particle. The origin of the coordinate is chosen to coincide with the bunch center in the steady state which is synchronized with the if phase. Assuming there are $M$ identical bunches in the ring, the $h$ th harmonic of the beam current $I_{h}$ is then given by

$$
I_{h}=I_{h}^{(0)}+I_{d c} \sum_{n=1}^{\infty} \frac{i^{n}}{n !}\left\langle\varphi^{n}\right\rangle_{p},
$$

where $I_{d c}=q M v_{0} N /(2 \pi R)$, is the averaged (dc) beam current, $v_{0}$ is the average particle speed, $i=\sqrt{-1}, N$ is the total number of particles in one bunch,

$$
\begin{aligned}
& I_{h}^{(0)}=\frac{I_{d c}}{N} \int_{-\pi R}^{\pi R} \int_{-\infty}^{\infty} e^{i h z / R} f_{0}\left(z, v_{z}\right) d v_{z} d z, \\
& \left\langle\varphi^{n}\right\rangle_{p}=\frac{R}{N h} \int_{-\varphi_{m}}^{\varphi_{m}} \int_{-\infty}^{\infty} \varphi^{n} f_{1}\left(z, v_{z}, t\right) d v_{z} d \varphi,
\end{aligned}
$$

is the $n$th moment due to the perturbation, $\varphi_{m}$ is the half-length of the bunch in the if phase, and use has been made of the relation $\varphi=h z / R$. The $n$th moment $\left\langle\varphi^{n}\right\rangle$ of the particle distribution is defined according to

$$
\left\langle\varphi^{n}\right\rangle=\frac{R}{N h} \int_{-\varphi_{m}}^{\varphi_{m}} \int_{-\infty}^{\infty} \varphi^{n} f\left(z, v_{z}, t\right) d v_{z} d \varphi,
$$

Assuming that $\left\langle\varphi^{n}\right\rangle=\left\langle\varphi^{n}\right\rangle_{0}+\left\langle\varphi^{n}\right\rangle_{1}$, i.e., $\left\langle\varphi^{n}\right\rangle$ consists of a steady part $\left\langle\varphi^{n}\right\rangle_{0}$ and a small perturbation part $\left\langle\varphi^{n}\right\rangle_{1}$; applying Eqs. (2.2) and (2.3), we can derive the following linearized equations for moments $\left\langle\varphi^{n}\right\rangle_{1}:[4]$

$$
\begin{gathered}
\frac{d^{2}\langle\varphi\rangle_{1}}{d t^{2}}+\omega_{s}^{2}\langle\varphi\rangle_{1}=\omega_{s}^{2}\left(\phi_{\nu}+\frac{\hat{V}}{V_{s}} \tan \psi_{s}\right), \\
\frac{d^{2}\left\langle\varphi^{2}\right\rangle_{1}}{d t^{2}}+4 \omega_{s}^{2}\left\langle\varphi^{2}\right\rangle_{1}=-2 \omega_{s}^{2} \varphi_{m}^{2}\left(\frac{\hat{V}}{V_{s}}-\phi_{v} \tan \psi_{s}\right),
\end{gathered}
$$

and

$$
\begin{aligned}
& \frac{d^{2}\left\langle\varphi^{m}\right\rangle_{1}}{d t^{2}}+m^{2} \omega_{s}^{2}\left\langle\varphi^{m}\right\rangle_{1}= \\
& m(m-1)\left\langle H_{0} \varphi^{m-2}\right\rangle_{0}\left\langle\varphi^{m-2}\right\rangle_{1} /\left\langle\varphi^{m-2}\right\rangle_{0} \\
& +m \omega_{s}^{2}\left[\phi_{v}+\left(\hat{V} / V_{s}\right) \tan \psi_{s}\right]\left\langle\varphi^{m-1}\right\rangle_{0} \\
& -m(m-1) \omega_{s}^{2} \varphi_{m}^{2}\left[\left(\hat{V} / V_{s}\right)-\phi_{v} \tan \psi_{s}\right]\left\langle\varphi^{m-2}\right\rangle_{0},
\end{aligned}
$$

for $m=2,3 \ldots$
The analysis is then proceeded further by identifying the perturbed moment $\left\langle\varphi^{n}\right\rangle_{1}$ in Eqs. (3.5)-(3.7) with $\left\langle\varphi^{n}\right\rangle_{p}$ in Eq. (3.1). The $h$ th harmonic of the beam current can be written in the polar coordinate notation as

$$
I_{h}=I_{b}(t) \mathrm{e}^{-i \phi_{b}(t)},
$$

where $I_{b}(t)=I_{b 0}+I_{b 1}, I_{b 0}=I_{h}^{(0)}, I_{b 1} \approx \sum_{j=1}^{\infty} I_{h, 2 j}^{(1)}$,

$$
\begin{gathered}
\phi_{b} \approx-\sum_{j=0}^{\infty} I_{h, 2 j+1}^{(1)} / I_{h}^{(0)}, \\
I_{h, 2 j}^{(1)}=(-1)^{j} I_{d c}\left\langle\varphi^{2 j}\right\rangle_{1} /(2 j) ! \\
I_{k, 2 j+1}^{(1)}=(-1)^{j} I_{d c}\left\langle\varphi^{2 j+1}\right\rangle_{1} /(2 j+1) !,
\end{gathered}
$$

and $I_{b 1}<<I_{b 0}$. If only the first two lowest moments (the dipole and the quadrupole modes) are retained in the expansion of $I_{b 1}$ and $\phi_{b}$, we have

$$
\begin{gathered}
I_{b 1} \approx I_{h, 2}^{(1)}=-I_{d c}\left\langle\varphi^{2}\right\rangle_{1} / 2, \\
\phi_{b} \approx-I_{h, 1}^{(1)} / I_{h}^{(0)} \approx-I_{d c}\langle\varphi\rangle_{1} / I_{h}^{(0)} .
\end{gathered}
$$

\section{THE EQUIVALENT CIRCUIT MODEL}

In the equivalent circuit model, an rf cavity is envisioned as a parallel $R L C$ circuit; the applied if power source and the circulating beam current are envisioned as currents $i_{g}$ and $i_{b}$, respectively. Using Kirchhoff's law, one can derive that the total voltage on the cavity satisfies the differential equation

$$
\frac{d^{2} v}{d t^{2}}+2 \alpha \frac{d v}{d t}+\omega_{r}^{2} v=2 \alpha R_{s} \frac{d}{d t}\left(i_{g}+i_{b}\right),
$$

where $v$ is the total voltage, $\alpha=\omega_{r} /(2 Q), Q$ is the quality factor of the cavity, $\omega_{r}$ is the resonant frequency of the cavity, and $R_{s}$ is the shunt resistance. Substituting

$$
i_{g}=I_{g} \exp \left\{-i\left(\omega_{g}+\psi_{g}\right) t\right\}
$$

$$
i_{b}=\left[I_{b 0}+I_{b 1}(t)\right] \exp \left\{-i\left[\omega_{g}+\phi_{b}(t)\right] t\right\},
$$

and

$$
v=\left[V_{s}+\hat{V}(t)\right] \exp \left\{-i\left[\omega_{g}+\psi_{v}+\phi_{v}(t)\right] t\right\},
$$

in Eq. (4.1), we can derive the following linearized equations for $\hat{V}$ and $\phi_{v}$ :

$$
\frac{d \hat{V}}{d t}+\alpha \hat{V}=-\alpha R_{s}\left[I_{b 1} \cos \psi_{\nu}+I_{b 0}\left(\phi_{b}-\phi_{\nu}\right) \sin \psi_{v}\right]
$$

and

$$
\begin{aligned}
& \frac{d \phi_{v}}{d t}+\alpha \phi_{\nu}=\frac{-\alpha R_{s} I_{b 0} \cos \psi_{\nu}}{V_{s}} \\
& \quad \times\left[\phi_{b}-\left(I_{b 1} / I_{b 0}\right) \tan \psi_{\nu}+\left(\hat{V} / V_{s}\right) \tan \psi_{v}\right]
\end{aligned}
$$


here $\omega_{g}$ is the frequency of the driving If, $\phi_{v}$ and $\phi_{b}$ are the phase deviations of $v$ and $i_{b}$ from their steady states, respectively. In arriving at Eqs. (4.2) and (4.3), we have assumed that the system is in tune, i.e. $\psi_{g}=\psi_{\nu}$, and we have chosen a rotating polar coordinate system such that the steady state beam current $I_{b 0}$ is on the real axis. Also, in deriving Eqs. (4.2) and (4.3), we have assumed that for high- $Q$ and high frequency resonators, $\alpha<<\omega_{g}$, and that the derivatives of the quantities $d\left[v \exp \left(i \omega_{g}\right)\right] / d t$ and $i_{b} \exp \left(i \omega_{g}\right)$ are negligible when compared, respectively, with the products of these quantities with $\omega_{\mathrm{g}}$. Eqs. (3.5), (3.6), (4.2) and 3 ) are the basic equations for studying the beam-cavity interaction.

\section{COMPARISON WITH KINETIC THEORY}

We now compare the dispersion relation derived from the moment method with that obtained from the Vlasov equation. We shall study the dispersion relations up to the second moment, or to the second harmonic of the synchrotron oscillation.

The complete set of equations, up to the second moment, are Eqs. (3.5), (3.6), (3.8), (3.9), (4.2) and (4.3). Making Laplace transformations to Eqs. (3.5), (3.6), (4.2) and (4.3); using Eqs. (3.8) and (3.9), one can derive a set of simultaneous linear algebraic equations for the unknowns $\tilde{\hat{V}}, \tilde{\phi}_{v}, \tilde{I}_{b 1}$, and $\tilde{\phi}_{b}$, where the symbol $\sim$ indicates the Laplace transformed quantities. Equating the determinant of the simultaneous algebraic equations to zero yields the dispersion relation:

$$
\begin{aligned}
s^{6} & +2 \alpha s^{5}+\left(5 \omega_{s}^{2}+\alpha^{2} \sec ^{2} \phi_{y}\right) s^{4} \\
& +10 \alpha \omega_{s}^{2} s^{3}+a_{2} s^{2}+8 \alpha \omega_{s}^{4} s+a_{0}=0,
\end{aligned}
$$

where $s$ is the Laplace transformation variable,

$$
\begin{aligned}
& a_{0}=4 \omega_{s}^{4}\left[\alpha^{2} \sec ^{2} \phi_{y}-\lambda^{2}\left(\mu_{1}+\mu_{2}\right)\right. \\
& \left.+\lambda^{2} \mu_{1} \mu_{2} \sec ^{2} \psi_{s}\right] \text {, } \\
& \dot{a}_{2}=4 \omega_{s}^{4}+5 \alpha^{2} \omega_{s}^{2} \sec ^{2} \phi_{y}-\omega_{s}^{2} \lambda^{2}\left(\mu_{1}+4 \mu_{2}\right), \\
& \lambda=\alpha R_{s} I_{b 0} / V_{s}, \mu_{1}=-I_{d c} / I_{b 0} \text {, and } \mu_{2}=I_{d c} \varphi_{m}^{2} /\left(4 I_{b 0}\right) \text {. } \\
& s^{6}+2 \alpha s^{5}+\left(5 \omega_{s}^{2}+\alpha^{2} \sec ^{2} \phi_{y}\right) s^{4} \\
& +10 \alpha \omega_{s}^{2} s^{3}+b_{2} s^{2}+8 \alpha \omega_{s}^{4} s+b_{0}=0,
\end{aligned}
$$

The derivation of the dispersion relation from the linearized Vlasov equation, including the second harmonic of the synchrotron frequency, has been presented in a previous work.[6] The result is quoted here in a slightly differ-

where

$$
\begin{gathered}
b_{0}=4 \omega_{s}^{4}\left\{\alpha^{2} \sec ^{2} \phi_{y}-\lambda^{2}\left[F_{1} \mu_{1}+\left(4 F_{2} / \varphi_{m}^{2}\right) \mu_{2}\right]\right. \\
\left.+\left(4 F_{1} F_{2} / \varphi_{m}^{2}\right) \lambda^{2} \mu_{1} \mu_{2} \sec ^{2} \psi_{s}\right\} \\
b_{2}=4 \omega_{s}^{4}+5 \alpha^{2} \omega_{s}^{2} \sec ^{2} \phi_{y} \\
\quad-\omega_{s}^{2} \lambda^{2}\left[F_{1} \mu_{1}+4\left(4 F_{2} / \varphi_{m}^{2}\right) \mu_{2}\right] \\
F_{m}=4 \int_{0}^{\infty}\left[J_{m}\left(\frac{h r}{R}\right)\right]^{2} \frac{d f_{0}}{d r} d r /\left(\frac{h}{R}\right)^{2} \int_{0}^{\infty} f_{0}(r) r d r
\end{gathered}
$$

is the reduced form factor [3], $r=\left[z^{2}+\left(v_{z} / \omega_{s}\right)^{2}\right]^{1 / 2}$, and $J_{l}(x)$ is the Bessel Function of the $l$ th order. The values of $F_{1}$ and $F_{2}$ have been calculated and charted for some different phase space distributions.[3] In general, the value of $F_{1}\left(F_{2}\right)$ decreases (increases) when the bunch length increases. For very short bunch lengths, $F_{1}$ and $F_{2}$ have values near 2.0 and 0 , respectively. At the bunching factor of 0.5 , most distributions have the values of $F_{1}$ near 1.3 and the values of $F_{2}$ near 0.17 . For short bunch length, $F_{2}$ is roughly proportional to $\varphi_{m}^{2}$.

Thus, by comparing Eqs. (5.1)-(5.3) with Eqs. (5.4)(5.6), we find that the dispersion relation derived from the equivalent circuit is the same as that inferred from the linearized Vlasov equation except for the factors $F_{1}$ and $4 F_{2} / \varphi_{m}^{2}$ which depend on the detail of the perturbation in the phase space.

\section{CONCLUSIONS}

We have formulated an approach, in the regime of linear approximation, to incorporate all harmonics of synchrotron oscillation in the equivalent circuit model of beam-cavity interaction by considering the perturbed moments of a bunched beam. We found good qualitative agreements in comparing the dispersion relations obtained from this new approach with that derived from the linearized Vlasov equation up to the second synchrotron harmonic.

\section{REFERENCES}

[1] K. W. Robinson, Report No. CEA-11, Cambridge Electron Accelerator, Cambridge, Mass. (Sept. 1956).

[2] K. W. Robinson, Report No. CEAL-101, Cambridge Electron Accelerator, Cambridge, Mass. (Feb. 1964).

[3] See, for example, T. F. Wang, Particle Accelerators, 54, pp. 105-126 (1990).

[4] T. F. Wang, Univ. of Tokyo, INS Report, INSRep.-1171, Nov. 1996.

[5] P. J. Channell, IEEE Trans. Nucl. Sci., 30 (4), Aug. 1983, p. 2607.

[6] W. P. Lysenko and M. Overley, Accelerators and Beam Optics Codes, AIP Conf. Proc. 177, ed. C. R. Eminhizer (AIP New York, 1988), p. 323. 